\title{
Red to green emitters from InGaP/InAlGaP laser structure by strain-induced quantum-well intermixing
}

\author{
A. A. Al-Jabr, M. A. Majid, C. Shen, T. K. Ng and B. S. Ooi* \\ Photonics Laboratory, King Abdullah University of Science \& Technology (KAUST) \\ Thuwal 23955-6900, Kingdom of Saudi Arabia (KSA)
}

*email: boon.ooi@kaust.edu.sa

\begin{abstract}
We increased the Al content in the single quantum well InGaP/InAlGaP laser by strain-induced quantum well intermixing, and obtained a considerable enhancement (close to ten-fold increase) in the photoluminescence (PL) intensity. Among the annealing process investigated, we achieved lasing at $638 \mathrm{~nm}$ in conjunction with reduction in the lasing threshold current by close to $500 \mathrm{~mA}$ in a moderately intermixed laser. Lasing in orange color, as well as spontaneous emission in the yellow and green color regime, were also achieved by extending the annealing conditions. The significance of the current work became apparent when one considers that achieving these tunable wavelengths by increasing the Al content in quantum wells during epitaxy growth leads to severe lattice-mismatch and poor material quality. Hence, our Al "drive-in" intermixing process is a viable approach for forming Al-rich InAlGaP quantum well, which is essential for realizing efficient optoelectronic devices in the "green-yellow-orange gap".
\end{abstract}

Keywords: AlGaInP laser diode, orange laser, intermixing, efficiency

\section{INTRODUCTION}

Optoelectronic devices, such as light emitting diodes (LEDs) and laser diodes (LDs) emitting in the green, yellow and orange suffer from low internal quantum efficiency [1], constituting the "green-gap". Hence commercial LDs in the wavelength range of $530 \mathrm{~nm}$ to $630 \mathrm{~nm}$ are largely inefficient or unavailable, although low efficiency LEDs are produced in this visible spectral region. The InAlGaP QWs having low $\mathrm{Al}$ content or Al-free are the dominant III-V quaternary/ternary alloys used for the growth of efficient optoelectronic devices in the visible spectrum towards in the red wavelength regime. Currently, the available commercial LDs have the shortest emission wavelength at $630 \mathrm{~nm}$, while the lowest reported wavelength by epitaxy growth is at $615 \mathrm{~nm}$ with Al content $\mathrm{y}=0.1$ [2]. The bandgap of $\mathbf{I n}_{\mathbf{x}}\left(\mathbf{A l}_{\mathbf{y}} \mathbf{G a}_{\mathbf{1 - y}}\right)_{1-\mathbf{x}} \mathbf{P}$ quantum well $(\mathrm{QW})$ can be tuned by controlling either the In content $(\mathrm{x})$ or the $\mathrm{Al}$ content $(\mathrm{y})$. For tuning the emission wavelength from red towards yellow, either $\mathrm{x}$ needs to be reduced (from $\mathrm{x}=0.48$ for lattice-matching to GaAs substrate), or $\mathrm{y}$ needs to be increased. However, the reduction in In content (x) led to increasing lattice-mismatch with GaAs substrates, rendering this option invalid.

The other option for reducing the emission wavelength is by increasing y in QW, and still maintaining lattice-matching condition. This is supposed to be the ideal solution for producing optoelectronic devices in the orange, yellow and green regime. However, introducing $\mathrm{Al}$ into the active region by the usual growth methods, such as metal-organic chemical vapor deposition (MOCVD), or molecular beam epitaxy (MBE), is accompanied by a reduction in efficiency. As a results, LEDs emitting in the yellow range utilizing Al-rich QWs typically employ 30 to 50 QWs stacks, and special fabrication techniques are utilized to improve light extraction [3].

Semiconductor Lasers and Laser Dynamics VII, edited by Krassimir Panajotov, Marc Sciamanna,

Angel Valle, Rainer Michalzik, Proc. of SPIE Vol. 9892, 98921B - (c) 2016 SPIE

CCC code: $0277-786 X / 16 / \$ 18 \cdot$ doi: $10.1117 / 12.2225164$

Proc. of SPIE Vol. 9892 98921B-1 
The issue related to the reaction of the background oxygen with $\mathrm{Al}$ during growth of the active region is non-trivial. The contamination of oxygen leads to oxygen-related DX-centers, forming deep-level traps in the QW, and thus increasing the nonradiative recombination centers and compromising radiative efficiency, which can be aggravated with increasing incorporation of $\mathrm{Al}$ [4]. Although growth at elevated temperature may lead to reduced oxygen-related defects, the elevated growth temperature beyond $780{ }^{\circ} \mathrm{C}$ does not favor In incorporation due to significant In evaporation [5], degrading the surface morphology as a result [6]. These inevitable effects does not allow further exploration into realizing the orange and yellow laser diodes using the InAlGaP alloy [7].

In our approach, instead of increasing $\mathrm{Al}$ during epitaxy growth, we employ QW intermixing (QWI) to achieve Al drive-in into the InGaP QWs, thus interdiffusing elements among barriers and QWs while achieving blueshift in the emission wavelength, as shown in Fig.3. Although QWI is a well-known process and had been studied intensively in numerous material systems [8-10], the progress for blueshifting the InAlGaP alloy for lasers application is currently lacking. There are mainly two types of QWI, i.e. the impurity induced disordering (IID) and the impurity-free vacancy disordering (IFVD). The available reports in the literature are related to the IID method [11, 12], in which the impurities introduced led to high defect density, rendering it suitable only for realizing passive section, such as the non-absorbing window (NAW) [5]. On the other hand, the IFVD is suitable for fabricating active devices as the defects introduced are significantly lower. Still the reports for IFVD on InAlGaP material system are limited [10,13,14]. In this work, we utilize our recently refined QWI process for increasing the Al content in QW, and achieved 10.5 times increase in PL intensity and marked improvement in the lasing characteristics of the fabricated devices, among others.

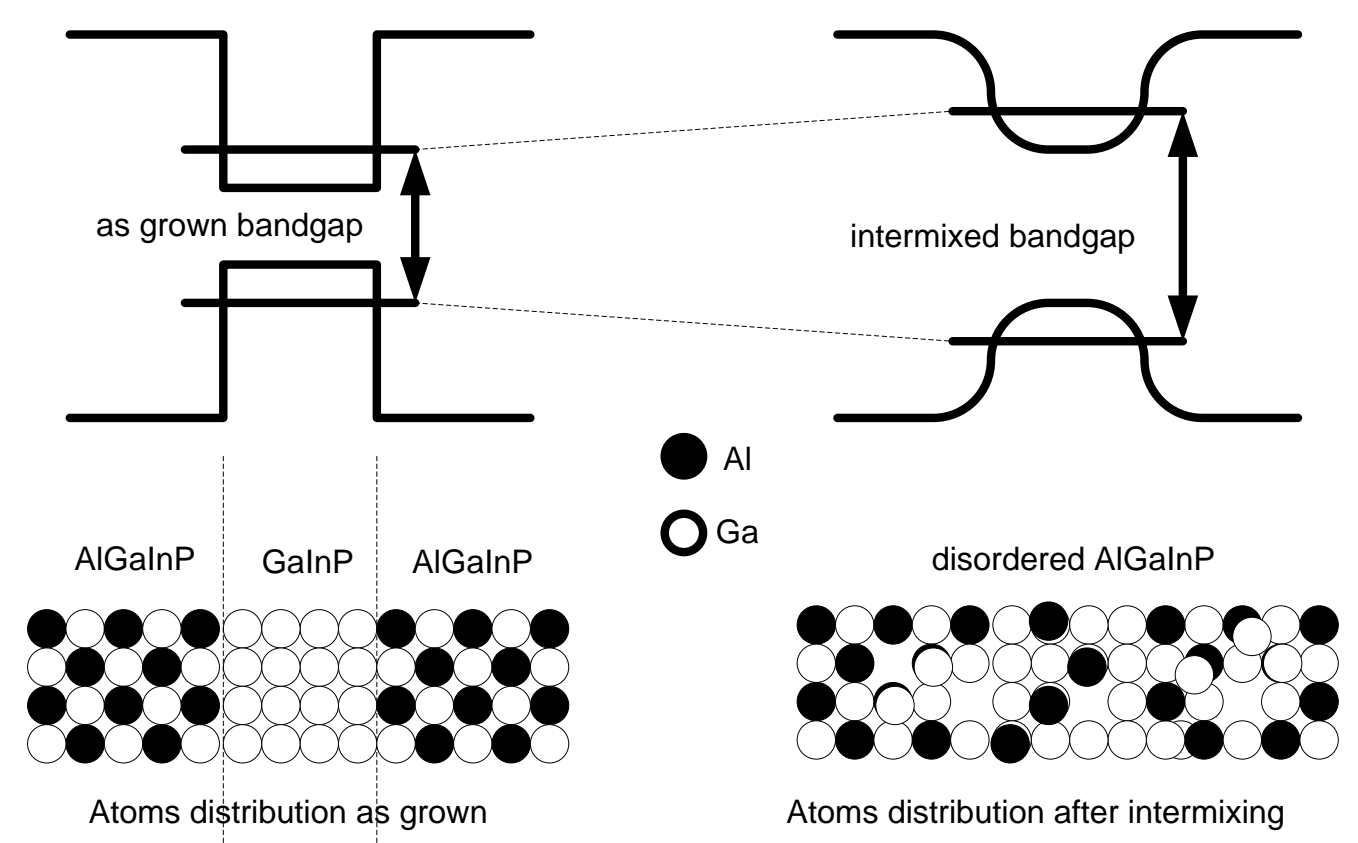

Fig. 1 Quantum well intermixing (QWI) process: the Al content in the QW is increased by diffusion causing the effective bandgap to increase. 


\section{EXPERIMENTS:}

The laser structure, as shown in Fig.2, has a single QW (SQW) InGaP/InAlGaP sandwiched between the $\mathrm{In}_{0.5} \mathrm{Al}_{0.5} \mathrm{P}$ cladding layers and GaAs contact layers, and grown on GaAs substrate. The process of intermixing includes depositing a $1 \mu \mathrm{m}$ thick $\mathrm{SiO}_{2}$ film that serves two purposes: the thick film forms a relatively high compressive strain on the top surface of the laser structure during annealing and at the same time protect the top surface during the process. After deposition, the structures are annealed to elevated temperatures from $900{ }^{\circ} \mathrm{C}$ to $1000{ }^{\circ} \mathrm{C}$ for durations from $20 \mathrm{~s}$ to $180 \mathrm{~s}$. The annealing process is repeated until the required wavelength is obtained, as described in detailed in [15]. The SQW structures were intermixed and then fabricated to laser devices. Laser sample were cleaved to approximately $1 \times 1 \mathrm{~cm}$. The blueshifts induced by the above procedure were measured at RT using micro-photoluminescence (PL) spectroscopy equipped with a $473 \mathrm{~nm}$ cobalt laser as the excitation source. The broad-area lasers with $75 \mu \mathrm{m}$ stripe-width were then fabricated from the intermixed samples, as well as the as-grown sample.

\begin{tabular}{|c|c|}
\hline [200 nm] & $\mathrm{p}$-GaAs $2 \times 10^{19}$ \\
\hline$[75 \mathrm{~nm}]$ & $\mathrm{p}-\ln _{0.5} \mathrm{Ga}_{0.5} \mathrm{P} 3 \times 10^{18}$ \\
\hline$[1000 \mathrm{~nm}]$ & $\mathrm{p}-\mathrm{In}_{0.5} \mathrm{Al}_{0.5} \mathrm{P} 1 \times 10^{18}$ \\
\hline$[80 \mathrm{~nm}]$ & $\mathrm{In}_{0.5} \mathrm{Al}_{0.3} \mathrm{Ga}_{0.2} \mathrm{P}$ Barrier \\
\hline$[6 \mathrm{~nm}]$ & $\mathrm{In}_{0.47} \mathrm{Ga}_{0.53} \mathrm{P} \quad \mathrm{QW}$ \\
\hline$[80 \mathrm{~nm}]$ & $\mathrm{In}_{0.5} \mathrm{Al}_{0.3} \mathrm{Ga}_{0.2} \mathrm{P}$ Barrier \\
\hline$[1000 \mathrm{~nm}]$ & $\mathrm{n}-\mathrm{In}_{0.5} \mathrm{Ga}_{0.5} \mathrm{P} 1 \times 10^{18}$ \\
\hline$[200 \mathrm{~nm}]$ & $\mathrm{n}-\mathrm{GaAs} 2 \times 10^{18}$ \\
\hline $\begin{array}{c}\text { n-GaAs } \\
\text { Substrate }\end{array}$ & GaAs Substrate \\
\hline
\end{tabular}

Fig. 2 The SQW laser structure.

\section{BANDGAP CALCULATIONS}

The change in emission wavelength can be related to the interdiffusion of $\mathrm{Al}$ and Ga between $\mathrm{QW}$ and barrier as follows.

$$
E g_{-} Q W=E g_{-} b u l k+E g_{-} Q+E g_{-} S
$$

where $\boldsymbol{E} \boldsymbol{g}_{-} \boldsymbol{Q W}$ is the bandgap of the QW, $\boldsymbol{E} \boldsymbol{g}_{-} \boldsymbol{b} \boldsymbol{u} \boldsymbol{l} \boldsymbol{k}$ is bandgap of the bulk material, $\boldsymbol{E} \boldsymbol{g}_{-} \boldsymbol{Q}$ is the bandgap due to quantum confinement and $\boldsymbol{E} \boldsymbol{g}_{-} \boldsymbol{S}$ is the bandgap due to strain. Here we are assuming the intermixing process has effect on interdiffusion of Al (x) and Ga (1-x) only, while In (y) content remained constant. The estimates help in understanding the bandgap change due to the effect of AlGa interdiffusion. To calculate $\boldsymbol{E} \boldsymbol{g}_{-} \boldsymbol{b u} \boldsymbol{l} \boldsymbol{k}, \boldsymbol{E} \boldsymbol{g}_{-} \boldsymbol{Q}$ and $\boldsymbol{E} \boldsymbol{g}_{-} \boldsymbol{S}$, we first calculate the lattice constant and the bandgap of the quaternaries of varying alloy contents. For the calculation of lattice constant, we linearly interpolated the lattice constants of the three binaries given in below: 
Table.1: The lattice constant values of the binary compound used in our calculations [16]

\begin{tabular}{|l|l|l|l|}
\hline Binary Compound & GaP & InP & AlP \\
\hline Lattice Constant $(\AA)$ & 5.4512 & 5.8688 & 5.4635 \\
\hline
\end{tabular}

For the given values of $x$ and $y$, the lattice constants of $\operatorname{In}_{x}\left(\mathrm{Al}_{y} \mathrm{Ga}_{1-y}\right)_{1-x} \mathrm{P}$ are calculated utilizing the constants given in Table. 1 as follows:

$$
\begin{gathered}
a\left(\operatorname{In}_{x} A l_{1-x} P\right)=x \cdot a(\operatorname{InP})+(1-x) \cdot a(A l P) \\
a\left(\operatorname{In} G a_{x} P\right)=x \cdot a(\operatorname{InP})+(1-x) \cdot a(G a P) \\
a\left(I_{x}\left(A l_{y} G a_{1-y}\right)_{1-x} P\right)=y \cdot a(\operatorname{In} A l P)+(1-y) \cdot a(\operatorname{InGaP})
\end{gathered}
$$

Similarly the bandgap can be estimated from the ternaries according to following equations [17, 18].

$$
\begin{gathered}
E_{g}\left(\operatorname{In}_{x} A l_{1-x} P\right)=1.351+1.83(1-x)+0.38(1-x)^{2} \\
E_{g}\left(I_{x} G a_{x} P\right)=1.351+0.643(1-x)+0.786(1-x)^{2} \\
E_{g}\left(I n_{x}\left(A l_{y} G a_{1-y}\right)_{1-x} P\right)=y \cdot E_{g}(\operatorname{In} A l P)+(1-y) \cdot E_{g}(I n G a P)
\end{gathered}
$$

Figure 2 shows the bandgap and lattice constants of InGaP as a function of In mole fraction. As we can see, the lattice constant is matched to GaAs substrate at approximately $x=0.48$. To reach the yellow wavelength, $\mathrm{x}=0.25$ is required, in which the ternary is not lattice matched to GaAs, and therefore this will degrade the QW crystal quality considerably.
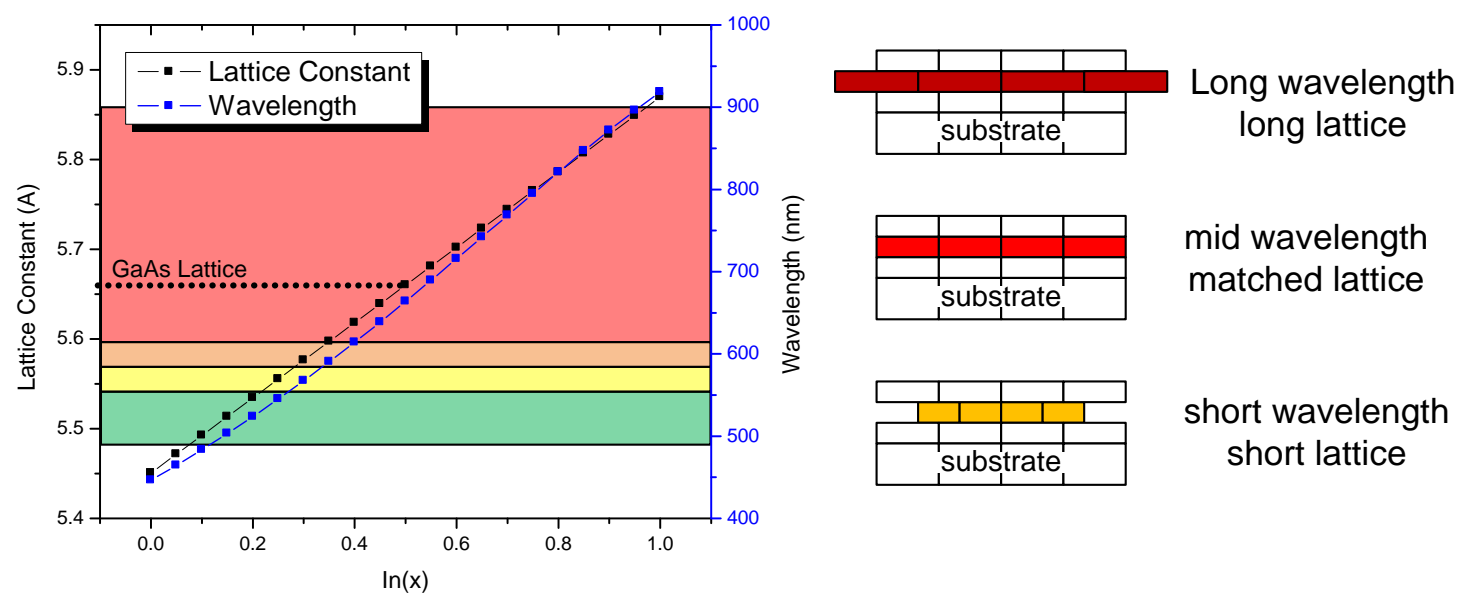

mid wavelength matched lattice

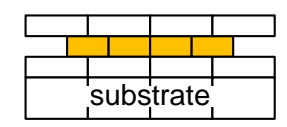

short wavelength short lattice

Fig. 3 The effect of changing In content on lattice constant and emission wavelength of InGaP, with the corresponding schematics showing the resultant lattice mismatch with respect to GaAs.

Figure 3 shows the emission wavelength and the lattice constants of InAlGaP active layer as a function of In content $(\mathrm{x})$. For our case, the In content $(\mathrm{x})$ is 0.47 , and the change in $\mathrm{Al}$ content $(\mathrm{y})$ alone during intermixing has insignificant effect on the lattice constant, though this varies the bandgap. At $y=0.45$, one could achieve yellow light emission. 

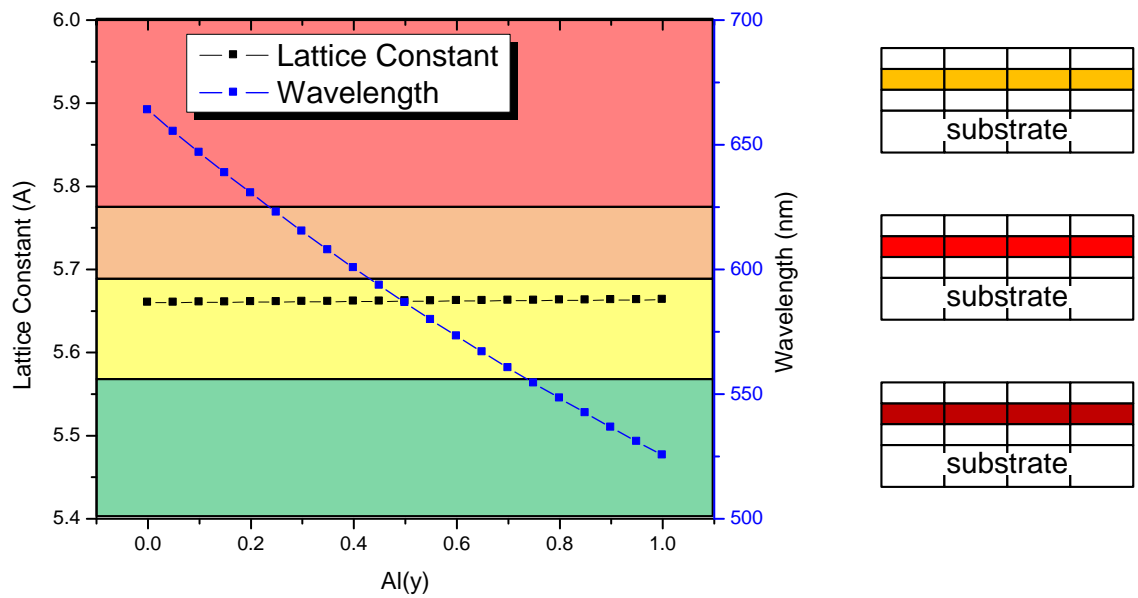

short wavelength

matched lattice

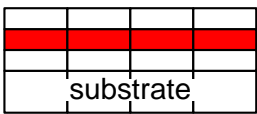

mid wavelength

matched lattice

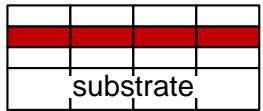

long wavelength matched lattice

Fig. 4 The effect of varying Al content on lattice constant and emission wavelength of InGaP. Reducing Al leads to the increases in wavelength, and the resultant alloys still maintain the lattice matching condition.

As can be seen from Fig.4, varying the $\mathrm{Al}$ content (y) has insignificant effect on the lattice constants of the InAlGaP quaternary alloys. Therefore, $\boldsymbol{E} \boldsymbol{g}_{-} \boldsymbol{S}$ does not change by increasing Al. The estimation of bandgap change neglects the effect of QW profile change on bandgap. A numerical model that takes error function profile into consideration would provide a rigorous treatment of the intermixing scenario. Nevertheless, the present estimates shed light on the effect of QWI on the Al drive-in process, which increases the $\mathrm{Al}$ content (y). The calculated $\boldsymbol{E} \boldsymbol{g}_{-} \boldsymbol{b} \boldsymbol{u} \boldsymbol{l k}$ of $\operatorname{In}_{0.47} \mathrm{Ga}_{0.53} \mathrm{P}$ is approximately $1.9116 \mathrm{eV}(650 \mathrm{~nm})$. Moving from bulk to QW, the bandgap increase by approximately $55 \mathrm{meV}$ to $1.967 \mathrm{eV}(630 \mathrm{~nm})$ due to the quantum confinement, with $\boldsymbol{E} \boldsymbol{g}_{-} \boldsymbol{Q}=\sim 80$ meV and $\boldsymbol{E} \boldsymbol{g}_{-} \boldsymbol{S} \sim-25 \mathrm{meV}$. The calculations of $\boldsymbol{E} \boldsymbol{g}_{-} \boldsymbol{Q}$ and $\boldsymbol{E} \boldsymbol{g}_{-} \boldsymbol{S}$ confinement and strain were taken from ref. [16], and not shown here. Figure 5 shows the bandgap and transition energy of bulk and 6 $\mathrm{nm} \mathrm{QW}$ for the $\mathrm{In}_{0.47}\left(\mathrm{Al}_{\mathrm{y}} \mathrm{Ga}_{1-\mathrm{y}}\right)_{0.53} \mathrm{P}$ alloy, respectively. The change in the peak emission wavelength is directly linked to the change in the constituent elements, and this is estimated to be $\sim 15-20 \mathrm{~nm}$ blueshift for 0.1 fraction change in $\mathrm{Al}$ content (y).

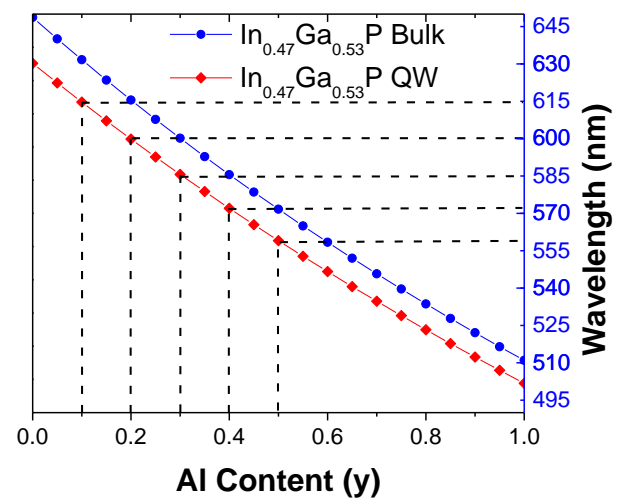

Fig. 5: The calculated peak emission wavelength of bulk and $6 \mathrm{~nm} Q W$ of $\operatorname{In}_{0.47}\left(A l_{y} G a_{1-y}\right)_{0.53} P$ alloy as the Al content (y) is increased. The change in $Q W$ profile and change in indium content due to intermixing are neglected for this calculation. 


\section{RESULTS:}

\section{A. INTERMIXING PROCESS}

Figure 6 shows the normalized PL spectra of the SQW laser samples after annealing at $950{ }^{\circ} \mathrm{C}$ for 30 $\mathrm{s}$, for $1-6$ cycles, respectively. The progression of peak wavelength blueshift with annealing cycle gives a rate of 6 to $5 \mathrm{~nm}$ blueshift per cycle, and each sample maintains good surface morphology after intermixing. As more annealing cycles are performed, the PL intensity gets weaker. This is the first repeatable and controllable QWI process that gives a high degree of intermixing and high crystal quality structure after intermixing, suitable for fabrication of tunable active devices.

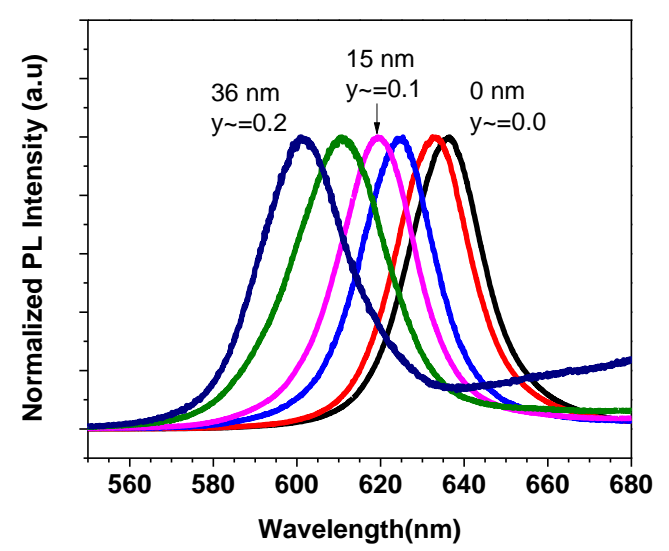

Fig. 6. The normalized PL spectra of SQW laser samples after annealing at $950{ }^{\circ} \mathrm{C}$ for 30 s for $1-6$ cycles.

\section{B. MODERATELY INTERMIXED DEVICES}

We investigate the effect of intermixing on the laser device performance after achieving $6 \mathrm{~nm}$ blueshift $(y \sim 0.03)$. Figure 7 (a) shows the PL of the sample before and after intermixing, which witness the enhancement of PL intensity by 10.5 times, while the full-wave at half-maximum (FWHM) was reduced from $33 \mathrm{~nm}$ to $20 \mathrm{~nm}$. The strong PL signal, narrow FWHM, and excellent surface morphology after the intermixing process suggest that the fabricated devices should operate at a reasonable performance. Figure 7(b) shows the lasing spectra of the fabricated devices as-grown and intermixed devices with 1-mm cavity length, fabricated in a single run. The devices were electrical pumped at 1.1 times the threshold current, $I_{\text {th }}$ and the lasing spectra were measured by placing an optical fiber close to the laser facets using translation stages. The as-grown laser devices emitted at $644 \mathrm{~nm}$ while the intermixed devices lasing wavelength was around $638 \mathrm{~nm}$. The small redshift between measured PL and EL of both intermixed and as-grown the peak emission is as expected.

Furthermore, we measured the threshold current ( $\left.\mathrm{I}_{\text {th }}\right)$ of several devices with 1-mm cavity length. The inset in Fig.7(b) shows the threshold current of the intermixed devices compared to that of the as grown. The significant advantage of our process is evidently reflected by the reduced threshold current, $\mathrm{I}_{\mathrm{th}}$, of $1.5 \mathrm{~A}$ in the intermixed laser, as compared to $2 \mathrm{~A}$ for that of the as-grown sample, an improvement by $25 \%$. The reduction in threshold current indicates that there is an improvement in 
the gain of the QW after annealing. The improvement in gain can be related to reduction of oxygenrelated defects as was reported in ref. [19].

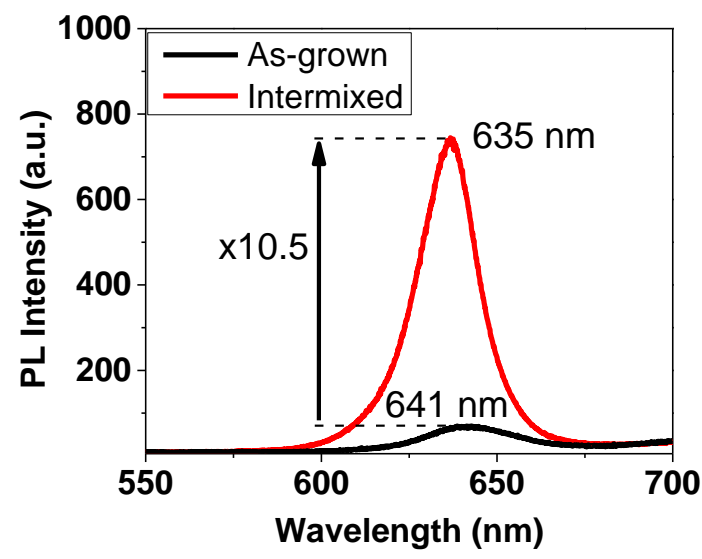

(a)

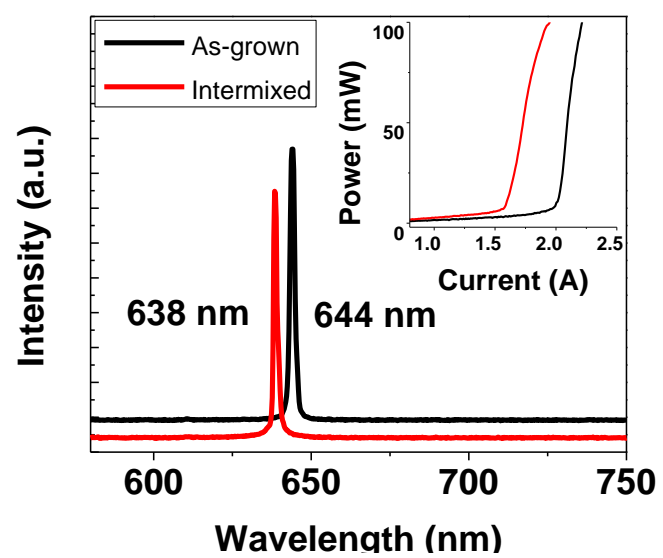

(b)

Fig. 7: (a) PL intensity of intermixed and as-grown samples, (b) lasing spectrum of as-grown and intermixed laser device with 1 mm long cavity. Inset in (b) compares the threshold current of the as-grown and intermixed laser structure. The intermixed device showed better threshold current, likely due to the reduction of oxygen-related DX centers in QWs, due to the elevated annealing temperature.

\section{HIGLY INTERMIXED DEVICES}

The same process described above was repeated for extended cycles to demonstrate devices emitting at orange and yellow wavelengths (see Fig. 8 and the corresponding insets). These devices emitted at orange $(620 \mathrm{~nm}$, a laser), and yellow (582 nm, a spontaneous emission device) after 5cycles and 9-cycles of annealing, respectively. The orange device lased at $608 \mathrm{~nm}$ with output power of $45 \mathrm{~mW}$ per facet while the yellow device emitted an output power of $\sim 2.5 \mathrm{~mW}$ through one of the facet. Even though the output power of the yellow device is low, it has comparable performance to commercially available LEDs, which may have up to $30 \mathrm{QW}$ stacks. We also demonstrated green emitters after 13- and 16-cycles of intermixing, albeit with low output power. This is expected as the alloy is approaching the indirect bandgap crossover of the $\Gamma-\mathrm{X}$ valleys. 


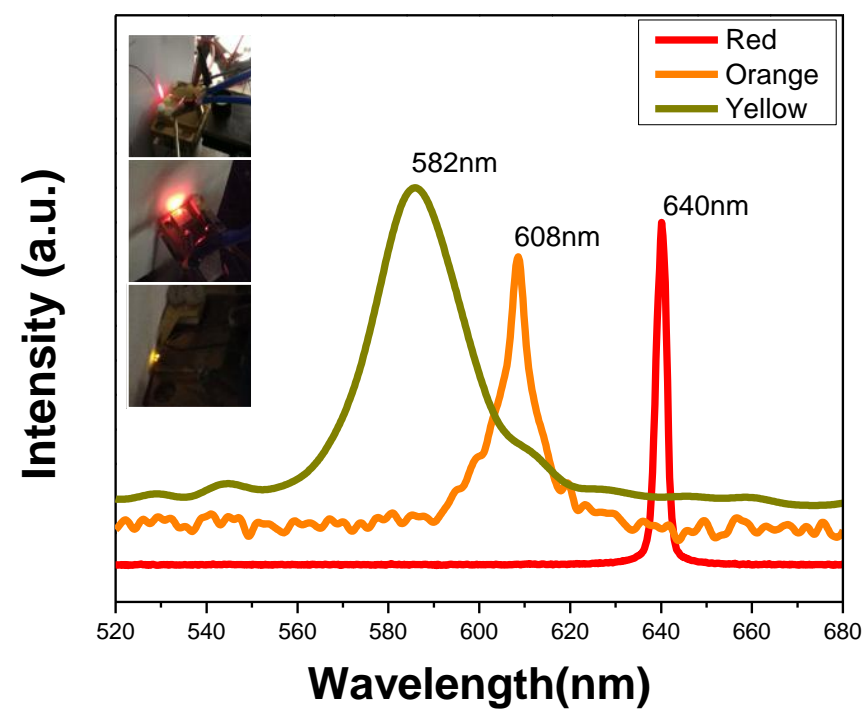

(a)

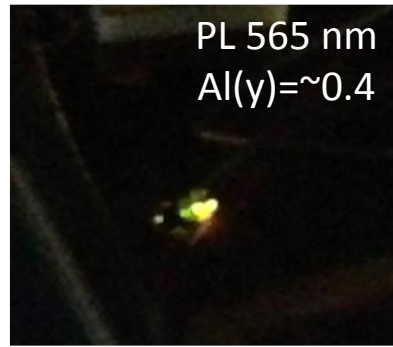

(b)

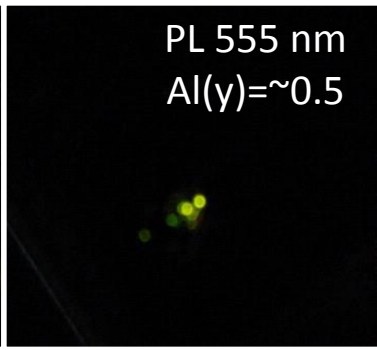

(c)

Fig. 8: (a) Intensity versus wavelength of as-grown (640 nm), and intermixed (608 $\mathrm{nm}$ and $582 \mathrm{~nm}$ ) devices with the corresponding images in the inset. The highly intermixed devices emitting at $565 \mathrm{~nm}$ (b) and $555 \mathrm{~nm}$ (c) were also demonstrated [19].

D. EFFECT OF INTERMIXING ON PL INTENSITY

Figure 9(a) shows the PL spectra of the as-grown and intermixed samples after removing the dielectric capping, The PL intensity of the intermixed samples is stronger than that of the as-grown. In this section, we converted the blueshift in wavelength into the corresponding change in $\mathrm{Al}$ content (y) based on the estimation model described in Section 2. We have noticed for a small QWI induced increment in $\mathrm{Al}$ content, the peak PL intensity increases rapidly before it starts to degrade progressively. The enhancement can be related to the removal of the oxygen-related defects by annealing while the degradation is likely due to the generation of point defects with increasing QWI cycles. Figure 9(b) compares between the PL intensity as Al content is increased by QWI and by growth. 


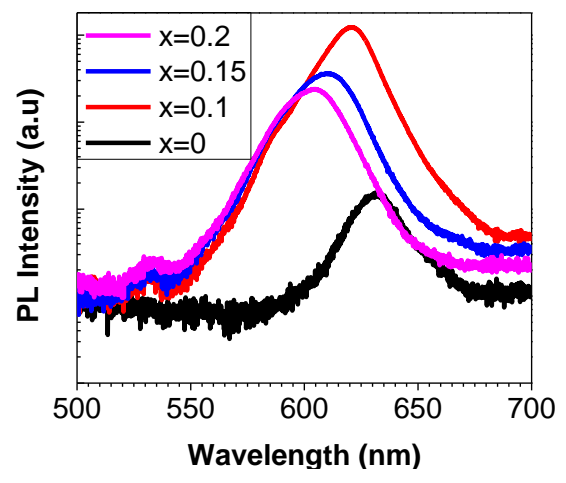

(a)

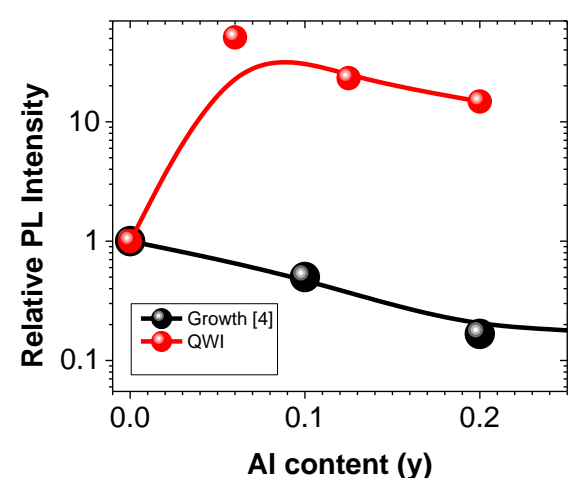

(b)

\begin{abstract}
Fig. 9 (a) The PL intensity versus wavelength with varying Al content, and (b) the relative PL intensity versus Al content for SQW laser structure achieved by epitaxy growth [11], and by post-growth QWI. The PL intensity is stronger when Al content is increased through QWI induced Al drive-in.
\end{abstract}

\title{
4. DISCUSSION
}

Extended QWI process is known to introduce defects in InGaP/InAlGaP laser structure. As a result, the intermixed devices may results in lower performance characteristics than that of the as-grown [20]. However, we achieved improvement in the InGaP/InAlGaP material system after annealing. Therefore, our results can be explained if we look at the QWI as three sub-processes. The first is the creation of defects, which are formed at the interface of the capping dielectric - laser interface, which propagates throughout the laser structure. The point defects are created due to the difference in expansion coefficients [21]. Defects created during QWI increase the non-radiative recombination centers, and also increase the optical losses for light propagation inside the laser structure. The defects density and cross-section can be controlled by reducing the annealing time or reducing the applied strain from the dielectric film. The second sub-process is the interdiffusion of group-III atoms between the QW and the barriers, resulting in bandgap blueshifting as more $\mathrm{Al}$ atoms diffuse into the QW with increasing temperature and/or time. The third process is the reduction of deep-level traps. The improvement in PL intensity and reduction in FWHM, in conjunction with the enhancement in threshold current $\mathrm{I}_{\text {th }}$ can be related to the reduction in deeplevel traps or oxygen-related DX centers [22].

\section{CONCLUSIONS}

We have increased the $\mathrm{Al}$ content in InGaP/InAlGaP laser structure by strain-induced $\mathrm{Al}$ drive-in QWI process. A moderately intermixed laser structure lased at $638 \mathrm{~nm}$ with a reduction in the lasing threshold current by close to $500 \mathrm{~mA}$, while a $620 \mathrm{~nm}$ orange color laser was achieved in highly intermixed laser structure. Our approach of increasing the Al content of InGaP/InAlGaP laser structure by QWI is therefore viable for fabrication of efficient emitters in the wavelengths below $630 \mathrm{~nm}$. 


\section{REFERENCES}

[1] A. OIDA, "LIGHT EMITTING DIODES (LEDS) FOR GENERAL ILLUMINATION," 2001.

[2] T. Tanaka, H. Yanagisawa, M. Takimoto, and S. Minagawa, "Tensile-strained AlGalnP singlequantum-well LDs emitting at 615 nm," Electronics Letters, vol. 29, pp. 1864-1866, 1993.

[3] H. J. Lee, Y. D. Cho, Y. J. Kim, C. H. Lee, J. H. Kim, H. S. Oh, et al., "Efficiency Improvement of 590 nm AlGalnP Light Emitting Diode with a Reflective Top Electrode," Journal of Physical Science and Application, vol. 4, 2014.

[4] S. Naritsuka, Y. Nishikawa, H. Sugawara, M. Ishikawa, and Y. Kokubun, "Photoluminescence studies on InGaAIP layers grown by low-pressure metalorganic chemical vapor deposition," Journal of electronic materials, vol. 20, pp. 687-690, 1991.

[5] R. Diehl, High-power diode lasers: fundamentals, technology, applications vol. 78: Springer Science \& Business Media, 2003.

[6] I. Yoshida, T. Katsuyama, and H. Hayashi, "Improvement of photoluminescence characteristics of AlGalnP double hetero-structures grown by OMVPE," Journal of crystal growth, vol. 115, pp. 533-537, 1991.

[7] G. B. Stringfellow and M. G. Craford, High Brightness Light Emitting Diodes: Academic Press, 1997.

[8] B. S. Ooi, K. Mcllvaney, M. W. Street, A. S. Helmy, S. G. Ayling, A. C. Bryce, et al., "Selective quantum-well intermixing in GaAs-AlGaAs structures using impurity-free vacancy diffusion," Quantum Electronics, IEEE Journal of, vol. 33, pp. 1784-1793, 1997.

[9] B.-S. Ooi, S. Ayling, A. Bryce, and J. Marsh, "Fabrication of multiple wavelength lasers in GaAsAlGaAs structures using a one-step spatially controlled quantum-well intermixing technique," Photonics Technology Letters, IEEE, vol. 7, pp. 944-946, 1995.

[10] K. Beernink, D. Sun, D. Treat, and B. Bour, "Differential Al-Ga interdiffusion in AlGaAs/GaAs and AlGalnP/GalnP heterostructures," Applied physics letters, vol. 66, pp. 3597-3599, 1995.

[11] K. Zheng, T. Lin, L. Jiang, J. Wang, S. Liu, X. Wei, et al., "High power red-light GalnP/AlGalnP laser diodes with nonabsorbing windows based on Zn diffusion-induced quantum well intermixing," Chinese Optics Letters, vol. 4, pp. 27-29, 2006.

[12] T. Lin, K. Zheng, C. Wang, and X. Ma, "Photoluminescence study of AlGalnP/GalnP quantum well intermixing induced by zinc impurity diffusion," Journal of Crystal Growth, vol. 309, pp. 140-144, 2007.

[13] J. Lie, Semiconductor Quantum Well Intermixing: Material Properties and Optoelectronic Applications vol. 8: CRC Press, 2000.

[14] C. Hamilton, O. Kowalski, K. Mcllvaney, A. Bryce, J. Marsh, and C. Button, "Bandgap tuning of visible laser material," Electronics Letters, vol. 34, pp. 665-666, 1998.

[15] A. A. Al-Jabr, M. A. Majid, M. S. Alias, D. H. Anjum, T. K. Ng, and B. S. Ooi, "Large bandgap blueshifts in the InGaP/InAlGaP laser structure using novel strain-induced quantum well intermixing," Journal of Applied Physics, vol. 119, p. 135703, 2016.

[16] L. A. Coldren, S. W. Corzine, and M. L. Mashanovitch, Diode lasers and photonic integrated circuits vol. 218: John Wiley \& Sons, 2012.

[17] D. Bour, J. Shealy, G. Wicks, and W. Schaff, "Optical properties of AlxIn1- xP grown by organometallic vapor phase epitaxy," Applied physics letters, vol. 50, pp. 615-617, 1987.

[18] H. J. Casey, Heterostructure lasers: Elsevier, 2012.

[19] J. Dekker, A. Tukiainen, N. Xiang, S. Orsila, M. Saarinen, M. Toivonen, et al., "Annealing of the deep recombination center in GalnP/AIGalnP quantum wells grown by solid-source molecular beam epitaxy," Journal of applied physics, vol. 86, pp. 3709-3713, 1999. 
[20] J. H. Marsh, P. Cusumano, A. C. Bryce, B. S. Ooi, and S. G. Ayling, "GaAs/AlGaAs photonic integrated circuits fabricated using impurity-free vacancy disordering," 1995, pp. 74-85.

[21] P. Gareso, M. Buda, L. Fu, H. Tan, and C. Jagadish, "Influence of SiO2 and TiO2 dielectric layers on the atomic intermixing of InxGa1- xAs/InP quantum well structures," Semiconductor Science and Technology, vol. 22, p. 988, 2007.

[22] P. Floyd and D. Treat, "Improved performance of laterally oxidized GalnP/AIGalnP lasers by thermal annealing," Applied physics letters, vol. 70, pp. 2493-2495, 1997. 